\title{
A Multi-agent System for Remote Eldercare
}

\author{
Boštjan Kaluža, Erik Dovgan, Violeta Mirchevska, Božidara Cvetković, Mitja \\ Luštrek, and Matjaž Gams
}

\begin{abstract}
This paper presents a case study in which a multi-agent system for care of the elderly people living at home alone is applied in order to prolong their independence. The system consists of several agents organized in groups providing robust and flexible monitoring, calling for help in the case of an emergency and issuing warnings if unusual behavior is detected. The first results and demonstrations show promising performance.
\end{abstract}

\section{Introduction}

Population aging in developed countries presents an increased pressure on the working-age population as well as on the national health budgets. A lot of elders cope with at least one health problem and many are dealing with two or more, e.g., hearth problems, arthritis, osteoporosis, cancer, frequent falls (which can lead to fractures), etc. Faced with decreased ability to function, they require either additional help or move to an eldercare facility. However, given the choice, elders mostly prefer to continue to live at home instead in an eldercare facility.

In this paper we present a case study of a multi-agent system that aims to prolong the independence of the elderly people living alone at their homes. The system is part of the Confidence project [5], which main objective is to build a remote care system for the elderly by detecting (i) short term disabilities such as falls and (ii) long term disabilities such as development of a disease. This paper presents the multi-agent architecture of the system and describes its prototype implementation.

The paper is organized as follows. Section 2 first presents a sample scenario as well as our vision how technology can promote confidence to both elders who live alone and their concerned family members, and second, it reviews the related work. Section 3 is devoted to description of the multi-agent architecture and its

Boštjan Kaluža, Erik Dovgan, Božidara Cvetković, Mitja Luštrek, and Matjaž Gams

Jožef Stefan Institute, Department of Intelligent Systems, Jamova 39, 1000 Ljubljana, Slovenia

Violeta Mirchevska

Result d.o.o., Bravničarjeva 11, 1000 Ljubljana, Slovenia

\{bostjan.kaluza, erik.dovgan, violeta.mircevska, boza.cvetkovic, mitja.lustrek, matjaz.gams\}@ijs.si 
components, while Section 4 describes prototype implementation and results. Finally, the last section outlines future work.

\section{Remote eldercare}

\subsection{Scenario}

Mrs. Brown, aged 71, lives in her house by herself since her husband passed away. Her grownup children moved to a different city and are able to visit her once or twice a month. Recently, Mrs. Brown tumbled on the floor while trying to clean the cabinet. She broke her left hip. After the recovery, she is able to prepare simple meals by herself, get dressed and take a bath or shower, but she feels unsecure and has an increased fear of falls. She avoids cleaning and walking around the house, and complains that she often feels dizzy. Although her doctor and children suggested several times, Mrs. Brown does not like the idea of moving from her home to an eldercare facility. In this situation, Mrs. Brown is living alone and despite not being at risk in terms of getting medical assistance there is a need for someone to take a look after her.

This aspect can be improved using remote eldercare technology, namely ambient intelligence including sensors, context-aware reasoning and on-line services. Now, let us imagine an updated scenario in which Mrs. Brown lives alone in a smart house under the care of the Confidence system. Her house is equipped with wireless sensors to monitor and analyze her posture and activities of daily living (ADL). The Confidence system is deployed to analyze her walking characteristics and detect any changes that might indicate a decreased performance, either related to her recent hip fracture or development of a new health problem. Confidence also monitors her activities of daily living in order to highlight deviations possibly correlated with illness or some other disability. In case Mrs. Brown suffers from a fall or just does not feel well, the Confidence system is trained to recognize even complex emergency situations and to call relatives or emergency center if needed. Overall, Mrs. Brown is still in her house, confident that if something goes wrong she will receive help even if she won't be able to make a call. Her concerned family members and doctor are now convinced that she can stay at home.

The primary goal of this research is to create a remote care system that can help enhance the quality of care for elders living in a smart environment by providing (i) detection of abnormal situations, meaning that the system is able to detect abnormal situations in short-term behavior, such as falls and loss of consciousness (in this case the system raises an alarm that results in phone call to family members or emergency center), and (ii) monitoring of behavior changes over longer periods of time in order to detect changes that indicate decreased performance. Gait analysis, for example, may discover gait disorders, which are an important indicator of a number of health problems: joint and muscular diseases, neurological disorders such as Parkinson's disease etc. Moreover, the goal is also 
to notify an increased danger of falling, which may appear with time, so that the user can start walking with a cane or a walker.

\subsection{Related Work}

Fall detection is one of the major challenges in eldercare, since falls are usually the main cause of hospitalization: older adults are five times more likely to be hospitalized for a fall-related injury than for any other cause [16] and moreover, fall injuries account for $40 \%$ of all nursing-home admissions [7]. Several systems were introduced to tackle fall discovery with accelerometers [6], [11], [19] and gyroscopes [2]. Pan et al. [15], for example, presented a homecare service for fall discovery using a body-worn tri-axial accelerometer and reporting such a discovery to an emergency center. Also, accelerometer-based fall detectors are already commercially available, for example, iLife ${ }^{\mathrm{TM}}$ [1] that employs panic button, accelerometers to detect falls and wireless transmitter, and Zenio [18], that provides similar product equipped with Bluetooth, so it can be connected to a PC or a mobile phone.

In recent years, agent-based systems have gained great importance in the healthcare field. Isern et al. [8] reviewed several agent-based solutions concluding that such an approach has positive affects in terms of modularity, efficiency, decentralization, flexibility, personalization, distributed planning, monitoring, proactivity and security. Luis et al. [3] presented the TeleCARE system, a multi-agent platform for virtual communities in elderly care. The main idea is to provide a framework upon which a variety of services can be employed. In addition, the project RoboCare [4] aims to develop a system consisting of a set of robotic and software agents to control the environment. The system tracks the elderly person with a camera, controls his or her daily activities and reports on any inconsistency in the execution.

Compared to the agent-based systems, which basically dealt with just one task, the objective of our research was twofold. The primary goal was to create a care system that is able to detect hazardous situations in short-term behavior (falls, loss of consciousness), which is one of the most common tasks in eldercare. The second, rarely addressed goal was to detect changes in behavior over longer periods of time. An important advantage of the system is that it is based on localization hardware (that enables context-depended reasoning resulting in a lower falsealarm rate). Localization enables a crucial advantage over accelerometer-based input because knowing that a person is in a bed enables avoiding annoying false alarms even when fast accelerations occur.

\section{System architecture}

The system is designed as a multi-agent system. Each system module, task or activity is designed as an agent, providing a service. Agents are organized into groups at a specific level of abstraction and coordinated by another, hierarchically 
higher-level agent. Each agent can be simply modified or replaced and new or redundant agents can be easily incorporated. An agent provides a service that is requested by another agent or is triggered by some event. Agents share their data through direct acquisition using three types of messages:

1. Measurement message is created when a new measurement is obtained from sensor agents. In this stage it contains only raw sensor data. The message is later augmented by other agents with additional interpretations, e.g. filtered/derived data, posture information, etc.

2. Action message is generated when an agent or an agent group requires service from another agent or group. Typical scenario is when a group of agents detect a fall and require alarm action from the communication agent group.

3. Status message is used to pool or post agent state.

The MAS architecture is illustrated in Figure 1. The main groups of agents and their interactions are presented with arrows.

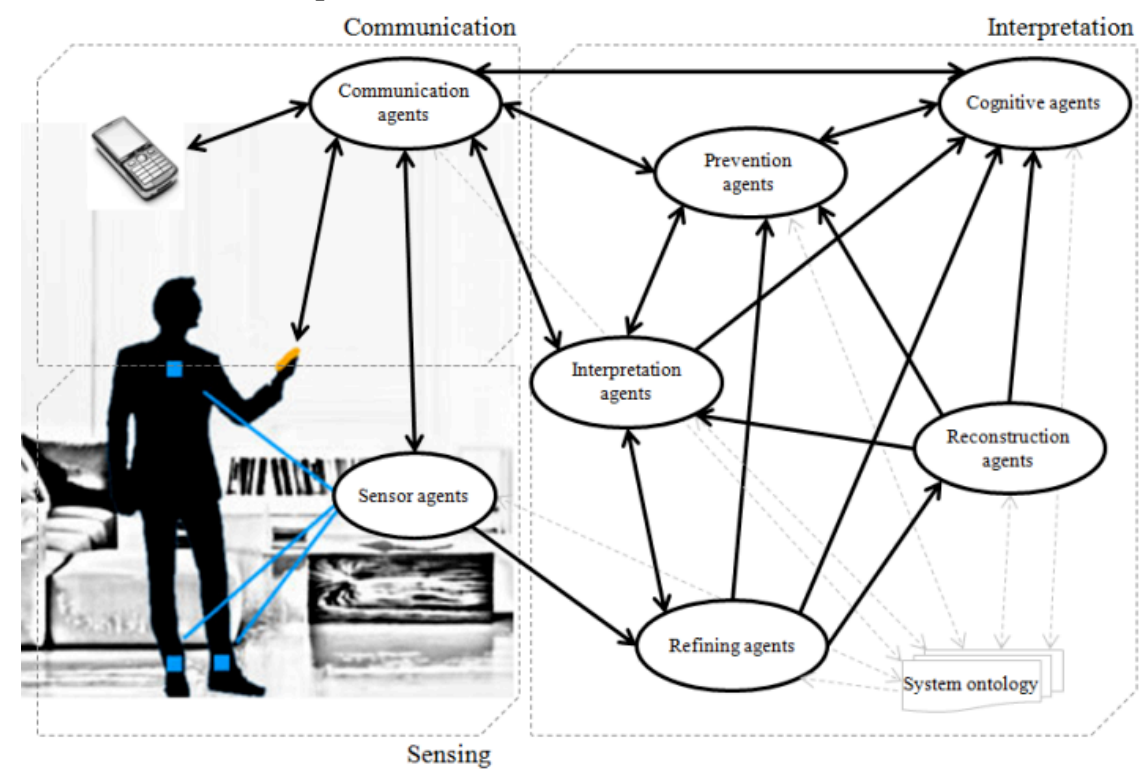

Fig. 1. Multi-agent architecture.

The architecture presented in Figure 1 contains seven groups of agents. Each group can contain several agents performing the same task (e.g., reading sensor data, recognizing posture) and some agents that coordinate final result of the group. At the lowest layer, an arbitrary location hardware system is employed. Sensing agents read raw sensor data, usually in sequential time periods $(1 / 10 \mathrm{sec}-$ ond), and serve them in a form of measurement message. At the end, a synchronization agent merges measurement messages within given time stamp to a single message. The sensor agents can also report other information, for example, a 
status message "low battery" is sent to communication agent group, which redistributes message to user and graphical interface.

The refining agents filter the noise, compute the derived attributes and map the raw data with the human-body model. As a result, the refining agent group augments the measurement message by providing a uniform representation of all available data of the body.

The reconstruction agents aim to determine the posture of the person in the environment. The group consists of classification agents based on machine learning (Random Forest) [12] and expert rules [14]. Coordination agent pulls a measurement message from the refining agent group and redistributes it to the classification agents. When all classification agents provide their label for the posture, the meta-classification agent merges (implemented with HMM) labels to the final posture classification.

The interpretation group of agents tries to detect if a person is in a dangerous situation. Consider the following situation as an example: an agent detects that the user is immovable, the reconstruction agents provide posture that the user is lying, and the refining agents give location 'kitchen'. The interpretation agents, implemented with data-driven (SVM) and knowledge-driven approach (expert rules), classify this situation as risky, since it is very unusual and most likely related to a health problem, e.g., the person might have lost consciousness, and inform the communication agent group.

The prevention-agent group monitors how the person behaves at various levels [13]. It consists of several agents observing a variety of statistics, e.g., walking characteristic, activity characteristics, daily dynamics [9], ADL, etc. Each agent pulls relevant measurement messages from other agents and builds its own behavior model (implemented with outlier detection). When an agent detects a deviation, it notifies group coordination agent, which decides upon notifying communication agents.

The next group is cognitive-state agent group: increasingly more abstract context awareness makes it possible to construct the cognitive state of the integrated system. The system design includes the cognitive state of the user, although not implemented yet in the tested version (all the other levels are already implemented). The goal is to reconstruct not only the physical, but also the cognitive state of the user and perform reasoning on a wider spectrum of information with an integrated reasoning strategy.

The last group consists of communication agents that are dedicated to user interaction, for example, agents that alert the user with a reply demand, make a phone call to relatives or help center, graphically display the state of the system, etc. An example of the communication agent is an alarm agent that triggers when a dangerous situation is reported by other agents and shuts down when the user responds that everything is OK. 


\section{Demonstration}

Our demonstration environment (a room) was organized as a near-realistic home apartment, equipped with a bed, a few chairs and tables, and divided into five logical areas: a kitchen; a sleeping area, a living area; a toilet; and a corridor. As localization component we selected the commercially available system Ubisense [17] that allows local positioning by tracking a set of small tags worn by a user. The tags were placed on chest, belt (optional), left and right ankles.

We have implemented a set of communication agents and graphical interface as depicted in Figure 2. The first three frames display the state of the reconstruction agent group: top perspective of the user in the room (frame 1); side view as coordinates $x$ and $z$ (frame 2); and the recognized activity by particular agents and joint prediction (frame 3). Next, the state of the interpretation-agent group is displayed in frame four with classification of situation. Frame five is dedicated to a live video stream (only for testing purposes, not part of the system). The agents from prevention group are displayed with histogram of movement (frame 6), a visualization of the daily-dynamic-analysis agent (frame 9) and other agents (frame 10). The status of the sensor agents and the available tags is presented in frame 7 , while the user-interaction agent (to cancel the raised alarm or trigger an alarm manually) is in frame 12. Currently, our prototype implementation is PC-based, while we are in the process of extending it with more advanced service-based agents such as an emergency call service, a portable device for alarm inhibition, a mobile phone, a remote web-based monitoring service, etc.

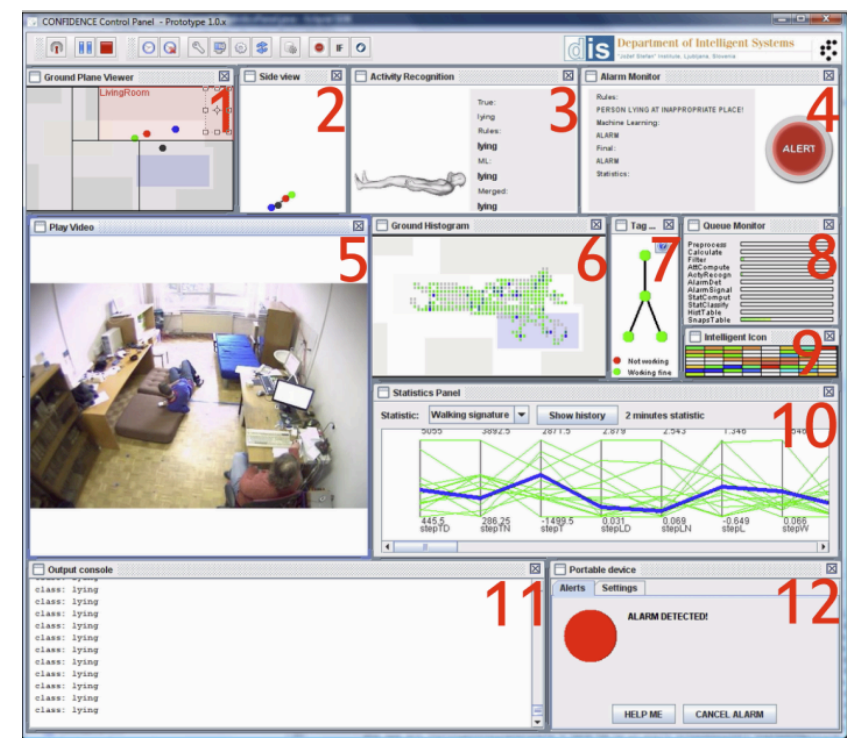

Fig. 2. The communication and graphical interface of the prototype system for eldercare. 
The system has been intensively tested in several complex scenarios where alarm must be raised and situations where false alarm is likely. The testes were performed in our testing room with young volunteers simulating walking, sitting, falling etc. The tests presented in [10] are shown in comparison with accelerometer-based system only and accelerometers with body orientation. The results in Table 1 show a significant difference, but one should have in mind that the cases were chosen to deal with situations that are hard to classify. More precisely, in a typical test, only simple laboratory cases are presented and publications typically report about $100 \%$ accuracy. However, in real life, $100 \%$ accuracy is not achieved. This is also the case with the Confidence since it was not able to detect with high accuracy if a person is sitting on a chair or on a floor in a time limit of 10 seconds. This is due to inaccurate position data that have standard deviation around $20 \mathrm{~cm}$. However, tripping, i.e., falling from walking, and fainting from standing, i.e., slowly lying down when fainting, were captured with $100 \%$ accuracy. Accelerometers with or without orientation are successful when dealing with fast falls, however, fail to recognize lying down slowly due to fainting and sliding from the chair. The last three rows in Table 2 denote cases when no alarm should be reported. In these cases, accelerometers with orientation perform better than accelerometers without orientation while the Confidence performs close to $100 \%$. Demo of this particular experiment with videos and results is available on http://dis.ijs.si/confidence/demo.html.

\begin{tabular}{lccc}
\hline Case / Accuracy [\%] & $\begin{array}{c}\text { Confidence } \\
\text { system }\end{array}$ & $\begin{array}{c}\text { Accelerometers and } \\
\text { orientation }\end{array}$ & $\begin{array}{c}\text { Only accelerome- } \\
\text { ters }\end{array}$ \\
\hline A: Tripping & 100.00 & 100.00 & 100.00 \\
A: Fainting standing & 100.00 & 0.00 & 0.00 \\
A: Sliding from the chair & 52.00 & 0.00 & 50.00 \\
\hline FA: Jumping in bed & 100.00 & 0.00 & 0.00 \\
FA: Sitting down quickly & 100.00 & 100.00 & 0.00 \\
FA: Searching for some- & 96.00 & 100.00 & 100.00 \\
thing under table/bed & $\mathbf{9 1 . 3 3}$ & $\mathbf{5 0 . 0 0}$ & $\mathbf{4 1 . 6 7}$ \\
\hline Overall & & &
\end{tabular}

Table 1. Performance evaluation of the Confidence system and systems using accelerometers.

\section{Conclusion}

In this paper we have presented a multi-agent system for remote eldercare that consists of several agents organized in groups that are able to detect falls and other situations potentially dangerous to the person. When compared to the accelerometers, the system achieved over $40 \%$ better performance. The Confidence project is in its final year, and is mainly devoted to in-depth testing and verification. How- 
ever, there are a couple of directions of current interest, e.g., the initialization procedure, user-friendly communication, and the cognitive-agent group.

Acknowledgments. This work was partly supported by the Slovenian Research Agency under the Research Programme P2-0209 Artificial Intelligence and Intelligent Systems, and partly from the EU's FP7/2007-2013, grant agreement No. 214986. The work of Violeta Mirchevska was partly financed by the European Union, European Social Found. We would like to thank Domen Marinčič, Rok Piltaver, Blaž Strle, and Damjan Kužnar for suggestions and discussions.

\section{References}

[1] AlertOne Services, Inc. iLife ${ }^{\mathrm{TM}}$ Fall Detection Sensor. http://www.falldetection.com.

[2] A.K. Bourke and G.M. Lyons, 'A threshold-based fall-detection algorithm using a bi-axial gyroscope sensor', Medical Engineering \&Physics, 30(1), 84 - 90, (2008).

[3] L. M. Camarinha-Matos and H. Afsarmanesh, 'A multi-agent based infrastructure to support virtual communities in elderly care', Int. Jour. of Networking and Virtual Organisations, 2(3), 246-266, (2004).

[4] Cesta and F. Pecora. Integrating Intelligent Systems for Elder Care in RoboCare. In W. C. Mann and A.Helal (Eds): Promoting Independence for Older Persons with Disabilities, IOS Press, pp. 65-73, 2006

[5] Confidence. http://www.confidence-eu.org, 2010-04-23.

[6] K. Doughty, R. Lewis, and A. McIntosh. The design of a practical and reliable fall detector for community and institutional telecare. Journal of Telemedicine and Telecare, vol. 6, pp. $150-154,2000$.

[7] G. F. Fuller. Falls in the elderly. American Family Physician. 61(7):2159-68,2173-4, 2000.

[8] D. Isern, D. Sanchez, A. Moreno, 'Agents applied in health care: A review', International Journal of Medical Informatics, 79(3), 145-166, 2010.

[9] B. Kaluža and M. Gams. An Approach to Analysis of Daily Living Dynamics. Proceedings of the WCECS 2010, vol. 1, pp. 485-490, ICMLDA'10, San Francisco, CA, October, 2010.

[10] B. Kaluža, V. Mirchevska, E. Dovgan, M. Lustrek, and M. Gams. An Agent-based Approach to Care in Independent Living. Lecture Notes in Computer Science, vol. 6439, pp. 177-186, AmI'10, Malaga, Spain, November, 2010.

[11] Kangas, M., Konttila, A., Winblad, I., and Jamsa, T. Determination of simple thresholds for accelerometry-based parameters for fall detection. In Proceedings of the 29th Conference of the IEEE, Engineering in Medicine and Biology Society, pp. 1367-1370, 2007.

[12] M. Luštrek and B. Kaluža, 'Fall detection and activity recognition with machine learning', Informatica, 33(2), 197-204, (2009).

[13] M. Luštrek, B. Kaluža, E. Dovgan, B. Pogorelc, and M. Gams, 'Behavior analysis based on coordinates of body tags', in AmI '09: Proceedings of the European Conference on Ambient Intelligence, pp. 14-23, Salzburg, Austria, (November 2009).

[14] V. Mirchevska, M. Luštrek, and M. Gams, 'Combining machine learning and expert knowledge for classifying human posture', in Proceedings of the Eighteenth International Electrotechnical and Computer Science Conference, volume B, pp. 183-186, (2009).

[15] Jiann-I Pan, Cheng-Jie Yung, Chung-Chao Liang, and Lien-Fu Lai, 'An intelligent homecare emergency service system for elder falling', in World Congress on Medical Physics and Biomedical Engineering 2006, pp. 424-428. Springer, (2006).

[16] Rubenstein LZ, Josephson KR, Robbins AS; Fall in the Nursing Home. Ann Intern Med. $121442-51,1994$.

[17] Ubisense. http://www.ubisense.net, 2010-04-23.

[18] Zenio, 'Zenio Fall Detector', http://www.zenio.be/product/8.html.

[19] T. Zhang, J. Wang, and J. Liu. 'Fall detection by wearable sensor and one-class SVM algorithm', in Lecture Notes in Control and Information Science, pp. 858-863, (2006). 